Research Article

\title{
Investigation of the Impact of the Pumping Beam Waist Size and Position on the Efficiency of YVO4/Nd : YVO4/YVO4 Laser Generation
}

\author{
Jaroslaw Mlynczak ${ }^{1}{ }^{1}$ and Maciej Zyskowski ${ }^{2}$ \\ ${ }^{1}$ Military University of Technology, Institute of Optoelectronics, Gen. S. Kaliskiego 2, Warsaw 00-908, Poland \\ ${ }^{2}$ Cisco Norway, Philip Pedersens Vei 1, Lysaker 1366, Oslo, Norway \\ Correspondence should be addressed to Jaroslaw Mlynczak; jaroslaw.mlynczak@wat.edu.pl
}

Received 29 April 2021; Revised 14 June 2021; Accepted 13 July 2021; Published 19 July 2021

Academic Editor: Sulaiman W. Harun

Copyright (C) 2021 Jaroslaw Mlynczak and Maciej Zyskowski. This is an open access article distributed under the Creative Commons Attribution License, which permits unrestricted use, distribution, and reproduction in any medium, provided the original work is properly cited.

This article presents the results of the investigation of the generation efficiency for different sizes and positions of the pumping beam waist inside the active medium of the $\mathrm{YVO}_{4} / \mathrm{Nd}$ : $\mathrm{YVO}_{4} / \mathrm{YVO}_{4}$ lasers. The measurements were carried out for a fixed resonator length of $36.1 \mathrm{~mm}$, a constant pumping power of $1.16 \mathrm{~W}$, and four output couplers with different radii of curvature. According to the knowledge of the authors, such an extended experimental approach is presented for the first time.

\section{Introduction}

Lasers based on the Nd:YVO4 active medium are an essential group of diode laser end-pumped solid-state lasers (DEPSSLs) due to their well-developed applications. Work on lasers with this active medium has been carried out for many years. This can be evidenced by numerous publications on this subject; however, in most cases, they relate to a specific laser configuration for a specific size and location of the pumping beam waist, which are usually close to the optimal values [1-11]. This article presents the results of the measurements of the generated beam power for different sizes and positions of the pumping beam waist for a constant resonator length of $36.1 \mathrm{~mm}$, a constant pumping power of $1.16 \mathrm{~W}$, and four output couplers with different radii of curvature. This may be a kind of complement to the work carried out so far. The measurement results were presented without theoretical analysis involving the matching of the pumping beam to the radiation distribution generated inside the resonator. Such an in-depth mathematical analysis may be the subject of further investigation on this type of lasers. However, this article can be successfully used as a reference by laser investigators and designers.

\section{Materials and Methods}

In the experiments, an active medium made by diffusion bonding of a neodymium-doped yttrium vanadate $(\mathrm{Nd}$ : $\mathrm{YVO}_{4}$ ) crystal of $10 \mathrm{~mm}$ length with undoped yttrium vanadate $\left(\mathrm{YVO}_{4}\right)$ of $2 \mathrm{~mm}$ length was used, as shown in Figure 1. The entire manufacturing and diffusion bonding process was carried out by Claser Photonics. The use of the undoped part of the medium at the ends of the laser rod significantly reduces the harmful effect of thermal phenomena, which, under unfavourable conditions, may lead to the resonator destabilization and, consequently, interruption of the laser action. Moreover, such a structure of the active medium protects it from thermal damage through better heat dissipation.

On one side of the active medium, a dichroic input mirror was deposited, which allowed for the introduction of the pumping beam into the optical cavity with minimal losses. The mirror was characterized by a high reflectivity of the generated radiation $(1064 \mathrm{~nm})$ and high transmission of the pumping radiation $(808 \mathrm{~nm})$. On the other side of the active medium, antireflective layers at the wavelength of $1064 \mathrm{~nm}$ were deposited to minimize the losses of the generated radiation. 


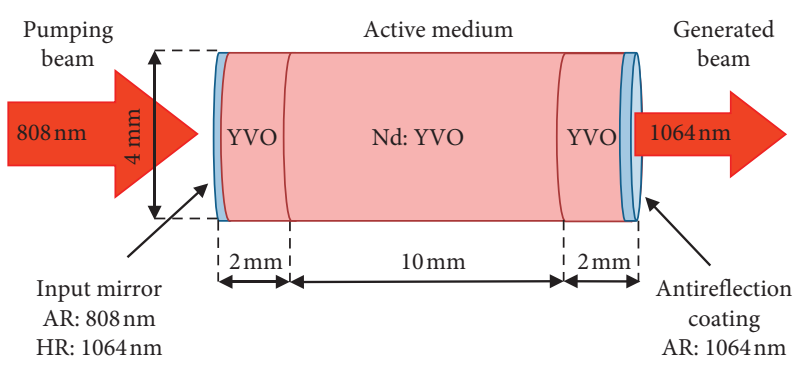

Figure 1: $\mathrm{YVO}_{4} / \mathrm{Nd}: \mathrm{YVO}_{4} / \mathrm{YVO}_{4}$ laser rod.

TABle 1: Parameters of the pumping beam.

\begin{tabular}{|c|c|c|c|c|}
\hline $\begin{array}{l}\text { Distance of the fibre from the } \\
\text { optics }(\mathrm{mm})\end{array}$ & $\begin{array}{c}\text { Diameter of the beam } \\
\text { waist }(\mu \mathrm{m})\end{array}$ & $\begin{array}{l}\text { Distance of the beam waist from } \\
\text { the optics }(\mathrm{mm})\end{array}$ & $\begin{array}{c}\text { Divergence of the beam } \\
(\mathrm{mrad})\end{array}$ & $\begin{array}{l}\text { Rayleigh range } \\
(\mathrm{mm})\end{array}$ \\
\hline 10 & 96 & 6 & 549 & 0.2 \\
\hline 7 & 118 & 8 & 393 & 0.3 \\
\hline 5 & 161 & 11 & 289 & 0.6 \\
\hline 3 & 252 & 16 & 181 & 1.4 \\
\hline 2 & 345 & 22 & 129 & 2.7 \\
\hline
\end{tabular}

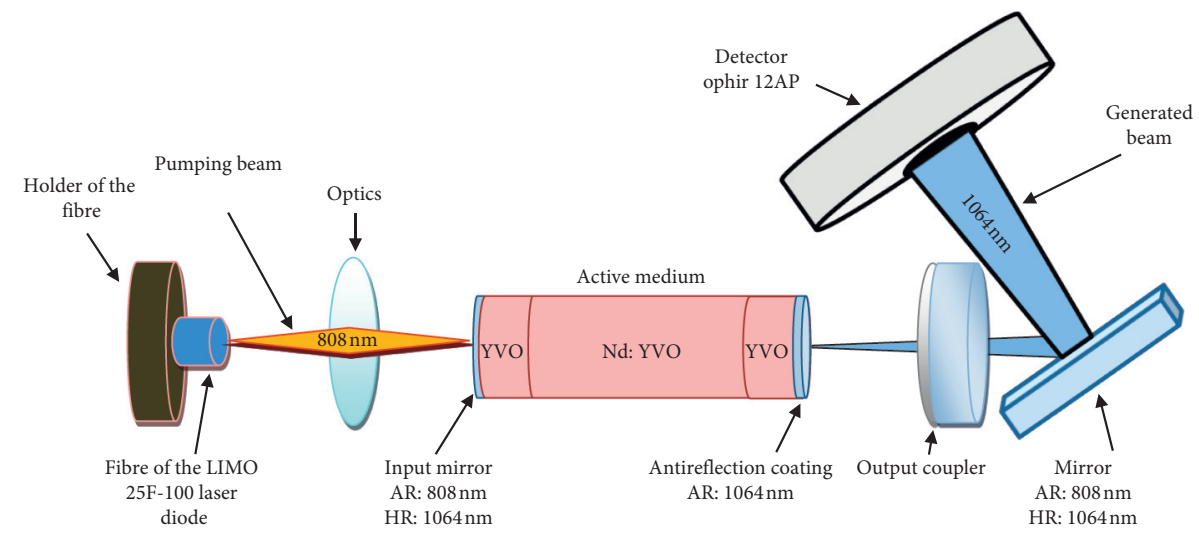

FIGURE 2: Experimental setup of the laser system.

The transmission of the active medium was measured using a Perkin Elmer Lambda 900 spectrometer in the range from $780 \mathrm{~nm}$ to $830 \mathrm{~nm}$. Based on the measured transmission, the absorption coefficient for the wavelength of $808 \mathrm{~nm}$ was determined in accordance with the formula $k=(1 / d) \ln (1 / T)$, where $d=10 \mathrm{~mm}$ and $T=6.8 \%$. Determined in this way, the absorption coefficient of $2.7 \mathrm{~cm}^{-1}$ corresponds to an absorption length of $0.37 \mathrm{~cm}$. On the basis of the absorption coefficient and the absorption cross section for a wavelength of $808 \mathrm{~nm}$ equal to $\sigma=6 \cdot 10^{-19} \mathrm{~cm}^{2}$ [12], the concentration of neodymium ions was determined according to the formula $N=k / \sigma$ and was equal to $N=4.5 \cdot 10^{18} \mathrm{~cm}^{-3}$.

The source of the pumping radiation was composed of a fibre coupled LIMO 25-F100 diode laser characterized by a $100 \mu \mathrm{m}$ core diameter and a 0.22 numerical aperture and a beam forming optic characterized by a $14 \mathrm{~mm}$ focal length. The spatial parameters of the pumping beam such as the waist, the distance of the waist from the optics, the divergence, and the Rayleigh range were changed by changing the distance of the fibre from the optics. The parameters of the pumping beam for a few distances of the fibre from the optics are presented in Table 1.

Figure 2 shows the experimental setup used to investigate the impact of the pumping beam waist size and position on the laser generation efficiency. For the experiments, the constant power of the pumping beam equal to $1.16 \mathrm{~W}$ (after passing through the optics) and the constant length of the resonator equal to $36.1 \mathrm{~mm}$ were applied. Four output couplers with different radii of curvature were used. The parameters of the output couplers are presented in Table 2. During the experiments, the active medium was air-cooled. To measure the power of the generated beam, it was necessary to use an appropriate filter separating the generated radiation $(1064 \mathrm{~nm})$ from the unabsorbed radiation of the pump $(808 \mathrm{~nm})$. For this purpose, an auxiliary dichroic mirror with high reflectance at $1064 \mathrm{~nm}$ and high transmission at $808 \mathrm{~nm}$ was used. The mirror was set so that the 
TABLe 2: Parameters of the output couplers.

\begin{tabular}{lcc}
\hline Output coupler & Radius of curvature $(\mathrm{mm})$ & Transmission at $1064 \mathrm{~nm}(\%)$ \\
\hline$R 50$ & 50 & 2.19 \\
$R 103$ & 103 & 2.25 \\
$R 150$ & 150 & 2.33 \\
$R \infty$ & $\infty$ & 4.36 \\
\hline
\end{tabular}

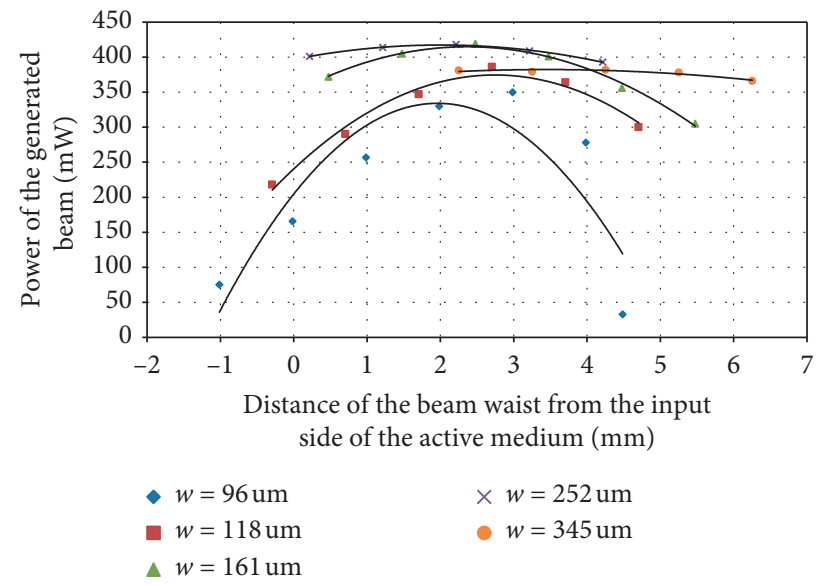

Figure 3: Power of the generated beam as a function of the distance of the beam waist from the input side of the active medium for the output coupler with the radius of $R=50 \mathrm{~mm}$.

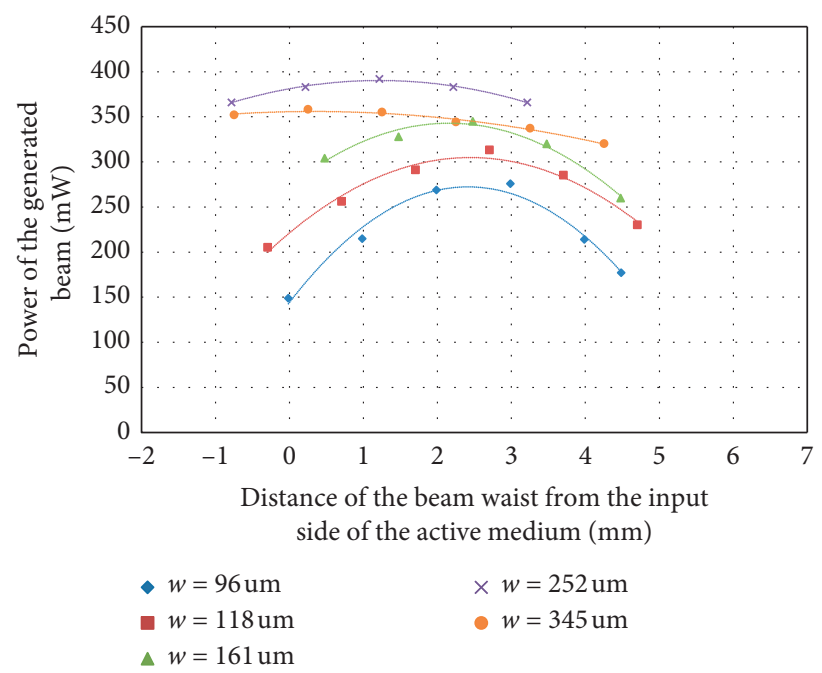

Figure 4: Power of the generated beam as a function of the distance of the beam waist from the input side of the active medium for the output coupler with the radius of $R=103 \mathrm{~mm}$.

angle of incidence of the radiation was minimal and did not exceed a few degrees.

\section{Results and Discussion}

The measurements of the dependence of the generated beam power on the pumping beam waist size and position for four different output couplers were carried out. Figures 3-6 show the generated beam power as a function of the pumping beam waist position at five different beam waists equal to $w=96 \mu \mathrm{m}, \quad w=118 \mu \mathrm{m}, \quad w=161 \mu \mathrm{m}, \quad w=252 \mu \mathrm{m}, \quad$ and $w=345 \mu \mathrm{m}$ for output couplers with a radius of curvature equal to $R=50 \mathrm{~mm}, R=103 \mathrm{~mm}, R=150 \mathrm{~mm}$, and $R=\infty \mathrm{mm}$, respectively.

In each case, the biggest changes in the output power as a function of the position of the beam waist were observed for the beam waist size equal to $96 \mu \mathrm{m}$. The bigger the waist, the smaller the changes of the power of the generated beam as a function of the waist position were measured. The highest power of the generated beam was recorded for a 


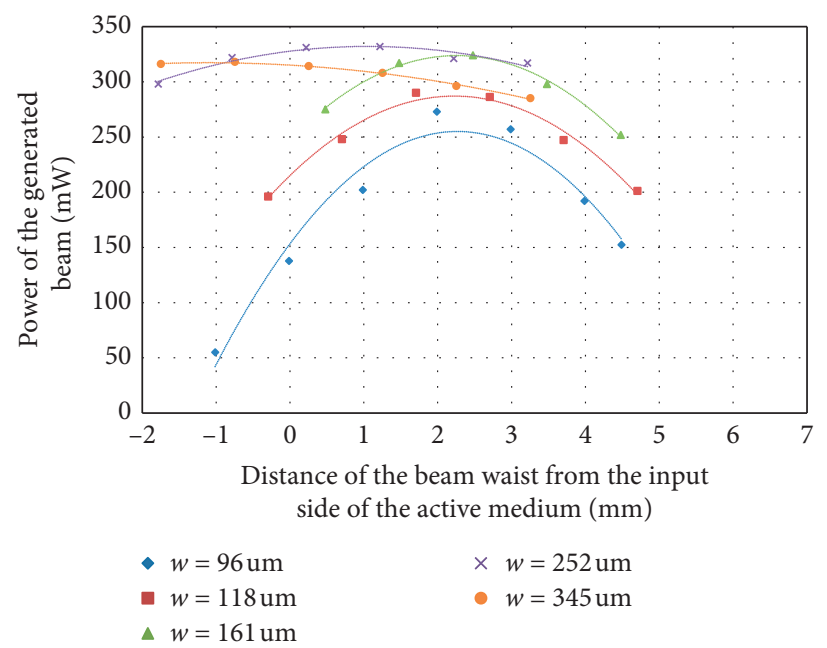

Figure 5: Power of the generated beam as a function of the distance of the beam waist from the input side of the active medium for the output coupler with the radius of $R=150 \mathrm{~mm}$.

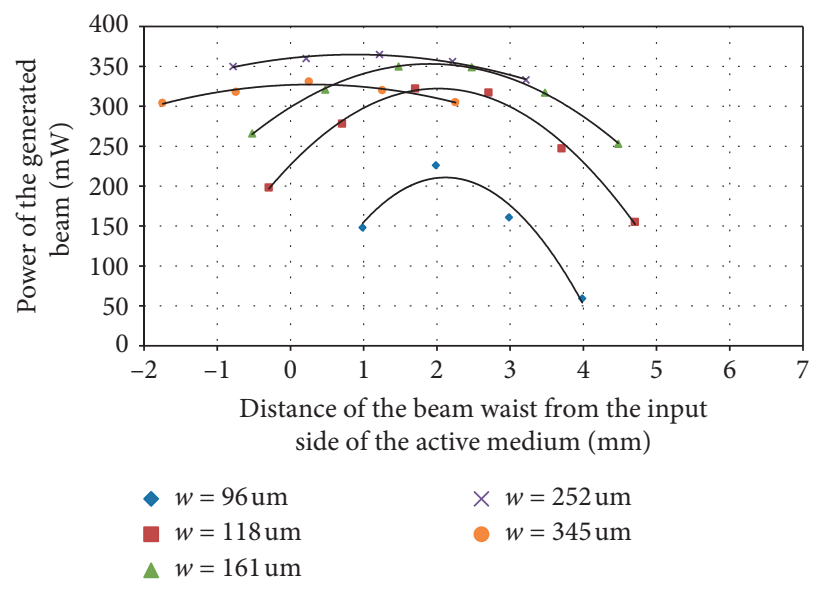

Figure 6: Power of the generated beam as a function of the distance of the beam waist from the input side of the active medium for the output coupler with the radius of $R=\infty \mathrm{mm}$.

pumping beam waist of $252 \mu \mathrm{m}$. The most effective, in terms of generation, positions of the beam waist for the $252 \mu \mathrm{m}$ waist and for different output couplers are shown in Table 3.

Taking into account all sizes of the pumping beam waist, it can be stated that the most effective position of the waist, in terms of generation, is approximately $2 \mathrm{~mm}$ from the input side of the active medium. This is the length of the undoped $\mathrm{YVO}_{4}$ crystal, so the beam waist is located at the input face of the neodymium-doped $\mathrm{Nd}$ : $\mathrm{YVO}_{4}$ crystal. For each of the four tested laser configurations, the pumping beam characterized by the waist of $252 \mu \mathrm{m}$ turned out to be the most effective with the highest power of the generated beam. The Rayleigh range of this pumping beam was $1.4 \mathrm{~mm}$. This means that, at a distance of $1.4 \mathrm{~mm}$ from the waist, the beam diameter was equal to $355 \mu \mathrm{m}$. The diameter of the active medium was $4 \mathrm{~mm}$; hence, it can be assumed that all pump radiations were absorbed by the active
TABLE 3: The most effective parameters of the pumping beam.

\begin{tabular}{lcc}
\hline Output coupler & Beam waist position $(\mathrm{mm})$ & Beam waist $(\mu \mathrm{m})$ \\
\hline$R 50$ & 2 & 252 \\
$R 103$ & 1.5 & 252 \\
$R 150$ & 1 & 252 \\
$R \infty$ & 1 & 252 \\
\hline
\end{tabular}

medium. The pumping beams with waists smaller than $252 \mu \mathrm{m}$ were characterized by a greater divergence angle and a shorter Rayleigh range. As a result, a significant part of the pumping radiation could escape beyond the active medium and did not participate in generation. In the case of the waist greater than $252 \mu \mathrm{m}$, the decrease in the generated power could be caused by too low power density in the active medium.

The presented effect can be explained by analysing the overlapping efficiency between the space characteristics of 
the pumping beam and the generated beam inside the resonator. However, such analysis may not be trivial because, for example, some thermal effects are difficult to model, especially if the input parameters are characterized by relatively high uncertainties. That is why the experimental results are very helpful.

\section{Conclusions}

The selection of the optimal spatial parameters of the pumping beam has a large impact on the generated power. During the investigation, it was found that, for the tested lasers based on the active medium $\mathrm{YVO}_{4} / \mathrm{Nd}: \mathrm{YVO}_{4} /$ $\mathrm{YVO}_{4}$, the most effective position of the waist, in terms of generation, was approximately $2 \mathrm{~mm}$ from the input side of the active medium. This is the length of the undoped $\mathrm{YVO}_{4}$ crystal, so the beam waist is located at the input face of the neodymium-doped $\mathrm{Nd}: \mathrm{YVO}_{4}$ crystal. The most efficient pumping beam was characterized by the waist of $252 \mu \mathrm{m}$

\section{Data Availability}

No data were generated or analysed in the presented research.

\section{Conflicts of Interest}

The authors declare no conflicts of interest regarding the publication of this paper.

\section{References}

[1] H. Zhang, J. Liu, J. Wang et al., "Laser properties of different Nd-doped concentration Nd:YVO4 laser crystals," Optics and Lasers in Engineering, vol. 38, no. 6, pp. 527-536, 2002.

[2] H. Zhnag, M. Chao, M. Gao, L. Zhang, and J. Yao, "High power diode single-end-pumped Nd:YVO4 laser," Optics and Laser Technology, vol. 35, no. 6, pp. 445-449, 2003.

[3] Z. Zhuang, T. Li, X. Li, and H. Yang, "Investigation of $\mathrm{Nd}$ : YVO4/YVO4 composite crystal and its laser performance pumped by a fiber coupled diode laser," Optics Communications, vol. 274, no. 1, pp. 176-181, 2007.

[4] F. J. Zhuang, Y. Q. Zheng, C. H. Huang, Y. Wei, H. Y. Zhu, and G. Zhang, "Efficient and compact intracavity-frequencydoubled YVO4/Nd:YVO4/KTP laser through analysis of the interaction length," Optics Communications, vol. 283, no. 17, pp. 3324-3327, 2010.

[5] A. F. El-Sherif and M. M. Talaat, "High efficient, high peak power of $18 \mathrm{~kW}$ with $4 \mathrm{~ns}$ pulses of diode pumped passive Q-switched and self Q-switched Nd: YVO4 laser at both $1064 \mathrm{~nm}$ using Cr:YAG and 532 nm using KTP crystals," Proceedings of SPIE, vol. 8599, Article ID 859927, 2013.

[6] S. Li, Y. Li, S. Zhao et al., "Thermal effect investigation and passively Q-switched laser performance of composite $\mathrm{Nd}$ : YVO4 crystals," Optics and Laser Technology, vol. 68, pp. 146-150, 2015.

[7] H.-Y. Lin, H. Liu, and Y.-P. Wang, "Comparative study of CW multi-wavelength Nd:GYSGG, Nd:YAG and Nd:YVO4 lasers at $1.3 \mu \mathrm{m}, " O p t i k$, vol. 147, pp. 123-127, 2017.

[8] O. M. Öztürk, M. M. Bayer, M. S. Anwar, M. Zakwan, and A. Serpengüzel, "Spectroscopy of a Nd:YVO4 diode pumped solid state laser," Microwave and Optical Technology Letters, vol. 59, no. 7, pp. 1636-1639, 2017.

[9] Z. Gao, Q. Feng, B. Yan et al., "Single longitudinal mode operation in diode-end-pumped wedge Nd:YVO4 laser," Optics Communications, vol. 424, pp. 131-136, 2018.

[10] G. Dudzik, K. Krzempek, and K. M. Abramski, "Comparison of Nd:YVO4-based monolithic microchip laser resonators for single-frequency stable, CW diode-pumped laser sources," 2019. Optics InfoBase Conference Papers, vol. Part F140CLEO_Europe 2019.

[11] Z. Gao, W. Zhang, B. Yan et al., "A tunable single-longitudinal-mode wedge Nd:YVO4 laser with a YVO4 wave-plate," Applied Physics B: Lasers and Optics, vol. 126, no. 11, 2020.

[12] https://www.rp-photonics.com/vanadate_lasers.html. 